Annals of Pure and Applied Mathematics

Vol. 21, No. 2, 2020, 129-133

ISSN: 2279-087X (P), 2279-0888(online)

Published on 23 May 2020

Annals of

www.researchmathsci.org

DOI: http://dx.doi.org/10.22457/apam.v21n2a8668

\title{
Some New Temperature Indices of Oxide and Honeycomb Networks
}

\author{
V.R.Kulli \\ Department of Mathematics \\ Gulbarga University, Gulbarga 585 106, India \\ E-mail: vrkulli@gmail.com
}

Received 2 May 2020; accepted 22 May 2020

Abstract. A graph index is a numerical parameter mathematically derived from the graph structure. In this paper, we introduce the first temperature index, modified first temperature index, temperature inverse degree, total temperature index, temperature zeroth order index, $F$-temperature index and general vertex temperature index of a graph. We compute these newly defined temperature indices for oxide networks and honeycomb networks.

Keywords: Temperature, first temperature index, $F$-temperature index, general vertex temperature index, oxide network, honeycomb network.

AMS Mathematics Subject Classification (2010): 05C05, 05C07, $05 C 12$

\section{Introduction}

A molecular graph or a chemical graph is a graph such that its vertices correspond to the atoms and the edges to the bonds. Chemical Graph Theory is a branch of Mathematical Chemistry. This branch of Mathematics has an important effect on the development of Science and Technology. Several graph indices have found many applications, especially, in QSPR/QSAR research, see $[1,2]$. Let $G$ be a finite, simple, connected graph. Let $V(G)$ and $E(G)$ denote the vertex set and edge set of $G$ respectively. The degree $d_{G}(v)$ of a vertex $v$ in $G$ is the number of vertices adjacent to $v$. For undefined term and notation, we refer the book [3].

The temperature of a vertex $u$ of a graph $G$ is defined by Fajtlowicz [5] as

$$
T(u)=\frac{d_{G}(u)}{n-d_{G}(u)},
$$

where $n$ is the number of vertices of $G$.

We introduce the following temperature indices.

The first temperature index of a graph $G$ is defined as

$$
T_{1}(G)=\sum_{u \in V(G)} T(u)^{2} .
$$

The modified first temperature index of a graph $G$ is defined as 


$$
{ }^{m} T_{1}(G)=\sum_{u \in V(G)} \frac{1}{T(u)^{2}} .
$$

The temperature inverse degree of a graph $G$ is defined as

$$
T I D(G)=\sum_{u \in V(G)} \frac{1}{T(u)} \text {. }
$$

The total temperature index of a graph $G$ is defined as

$$
T T(G)=\sum_{u \in V(G)} T(u)
$$

The temperature zeroth order index of a graph $G$ is defined as

$$
T Z(G)=\sum_{u \in V(G)} \frac{1}{\sqrt{T(u)}} .
$$

The $F$-temperature index of a graph $G$ is defined as

$$
F T(G)=\sum_{u \in V(G)} T(u)^{3}
$$

The general vertex temperature index of a graph $G$ is defined as

$$
T_{1}^{a}(G)=\sum_{u \in V(G)} T(u)^{a}
$$

where $a$ is a real number.

Recently, some temperature indices were introduced and studied, for example, in $[5,6,7,8,9]$. Recently, some graph indices were studied in $[10,11,12,13,14]$. In this paper, the first temperature index, modified first temperature index, temperature zeroth order index, $F$-temperature index, general vertex temperature index for oxide and honeycomb networks are computed.

\section{Results for Oxide networks}

Oxide networks are of vital importance in the study of silicate networks. An Oxide network of dimension $n$ is denoted by $O X_{n}$. A 5-dimensional oxide network is shown in Figure 1.

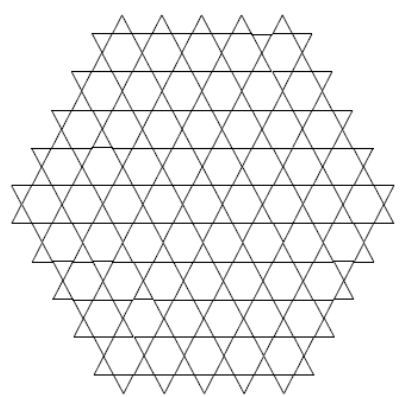

Figure 1: A 5-dimensional oxide network

Theorem 1. The general vertex temperature index of an oxide network $O X_{n}$ is given by

$$
T_{1}^{a}\left(O X_{n}\right)=6 n\left(\frac{2}{9 n^{2}+3 n-2}\right)^{a}+\left(9 n^{2}-3 n\right)\left(\frac{4}{9 n^{2}+3 n-4}\right)^{a} \text {. }
$$


Some New Temperature Indices of Oxide and Honeycomb Networks

Proof: Let $G$ be the graph of oxide network $O X_{n}$. Clearly the vertices of $O X_{n}$ are either of degree 2 or 4 , see Figure 1. By calculation, we obtain that $G$ has $9 n^{2}+3 n$ vertices and $18 n^{2}$ edges. We partition $V(G)$ into two sets, vertices of degree 2 and 4 respectively.

$$
\begin{aligned}
& V_{1}=\left\{u \in V(G) \mid d_{G}(u)=2\right\}, \quad\left|V_{1}\right|=6 n . \\
& V_{2}=\left\{u \in V(G) \mid d_{G}(u)=4\right\}, \quad\left|V_{2}\right|=9 n^{2}-3 n .
\end{aligned}
$$

Therefore we obtain the vertex partition based on the temperature of the vertices as given in Table 1.

$$
\begin{array}{ccc}
T(u) \backslash u \in V(G) & \frac{2}{9 n^{2}+3 n-2} & \frac{4}{9 n^{2}+3 n-4} \\
\text { Number of edges } & 6 n & 9 n^{2}-3 n
\end{array}
$$

Table 1: Vertex partition of $O X_{n}$

By definition, we have $T_{1}^{a}(G)=\sum_{u \in V(G)} T(u)^{a}$. Thus by using Table 1, we deduce

$$
T_{1}^{a}\left(O X_{n}\right)=6 n\left(\frac{2}{9 n^{2}+3 n-2}\right)^{a}+\left(\frac{4}{9 n^{2}+3 n-4}\right)^{a} .
$$

From Theorem 1, we obtain the following results.

Corollary 1.1. Let $O X_{n}$ be an oxide network of dimension $n$. Then

$$
\begin{aligned}
& T_{1}\left(O X_{n}\right)=6 n\left(\frac{2}{9 n^{2}+3 n-2}\right)^{2}+\left(9 n^{2}-3 n\right)\left(\frac{4}{9 n^{2}+3 n-4}\right)^{2} . \\
& { }^{m} T_{1}\left(O X_{n}\right)=\frac{1}{16}\left[24 n\left(9 n^{2}+3 n-2\right)^{2}+\left(9 n^{2}-3 n\right)\left(9 n^{2}+3 n-4\right)^{2}\right] . \\
& T I D\left(O X_{n}\right)=\frac{1}{4}\left[12 n\left(9 n^{2}+3 n-2\right)+\left(9 n^{2}-3 n\right)\left(9 n^{2}+3 n-4\right)\right] . \\
& T T\left(O X_{n}\right)=\frac{12 n}{9 n^{2}+3 n-2}+\frac{4\left(9 n^{2}-3 n\right)}{9 n^{2}+3 n-4} . \\
& T Z\left(O X_{n}\right)=6 n\left(\frac{2}{9 n^{2}+3 n-2}\right)^{\frac{1}{2}}+\left(9 n^{2}-3 n\right)\left(\frac{4}{9 n^{2}+3 n-4}\right)^{\frac{1}{2}} \\
& F T\left(O X_{n}\right)=6 n\left(\frac{2}{9 n^{2}+3 n-2}\right)^{3}+\left(9 n^{2}-3 n\right)\left(\frac{4}{9 n^{2}+3 n-4}\right)^{3}
\end{aligned}
$$

Proof: Put $a=2,-2,1,-1,-1 / 2,3$ in equation (i), we obtain the desired results.

\section{Results for Honeycomb networks}

Honeycomb networks are very useful in chemistry and also in computer graphics. A honeycomb network of dimension $n$ is denoted by $H C_{n}$, where $n$ is the number of hexagons between central and boundary hexagon. A 4-dimensional honeycomb network is presented in Figure 2. 


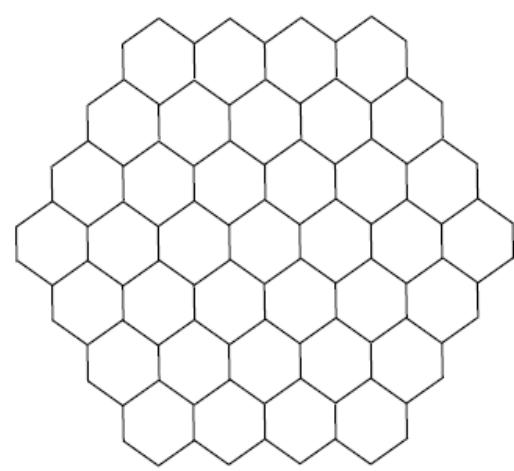

Figure 2: A 4-dimensional honeycomb network

Theorem 2. The general vertex temperature index of a honeycomb network $H C_{n}$ is

$$
T_{1}^{a}\left(H C_{n}\right)=6 n\left(\frac{1}{3 n^{2}-1}\right)^{a}+\left(6 n^{2}-6 n\right)\left(\frac{1}{2 n^{2}-1}\right)^{a} .
$$

Proof: Let $H$ be the graph of honeycomb network $H C_{n}$. The vertices of $H C_{n}$ are either of degree 2 or 3, see Figure 2. By calculation, we obtain that $H$ has $6 n^{2}$ vertices and $9 n^{2}-3 n$ edges. We partition the vertex set of $H$ into two sets, vertices of degree 2 and 3 respectively.

$$
\begin{array}{lll}
V_{1}=\left\{u \in V(H) \mid d_{H}(u)=2\right\}, & & \left|V_{1}\right|=6 n . \\
V_{2}=\left\{u \in V(H) \mid d_{H}(u)=3\right\}, & & \left|V_{2}\right|=6 n^{2}-6 n .
\end{array}
$$
follows:

Therefore we find the vertex partition based on the temperature of the vertices as

$$
\begin{array}{ll}
T V_{1}=\left\{u \in V(H) \mid T(u)=\frac{2}{6 n^{2}-2}\right\}, & \left|T V_{1}\right|=6 n . \\
T V_{2}=\left\{u \in V(H) \mid T(u)=\frac{3}{6 n^{2}-3}\right\}, & \left|T V_{2}\right|=6 n^{2}-6 n .
\end{array}
$$

By definition, we have $T_{1}^{a}(H)=\sum_{u \in V(H)} T(u)^{a}$. Thus

$$
\begin{aligned}
T_{1}^{a}\left(H C_{n}\right) & =\left|T V_{1}\right|\left(\frac{2}{6 n^{2}-2}\right)^{a}+\left|T V_{2}\right|\left(\frac{3}{6 n^{2}-3}\right)^{a} \\
& =6 n\left(\frac{1}{3 n^{2}-1}\right)^{a}+\left(6 n^{2}-6 n\right)\left(\frac{1}{2 n^{2}-1}\right)^{a}
\end{aligned}
$$

Corollary 2.1. Let $H C_{n}$ be a honeycomb network of dimension $n$. Then
(1) $T_{1}\left(H C_{n}\right)=\frac{6 n}{\left(3 n^{2}-1\right)^{2}}+\frac{6 n^{2}-6 n}{\left(2 n^{2}-1\right)^{2}}$.
(2) ${ }^{m} T_{1}\left(H C_{n}\right)=6 n\left(3 n^{2}-1\right)^{2}+\left(6 n^{2}-6 n\right)\left(2 n^{2}-1\right)^{2}$.
(3) $T I D\left(H C_{n}\right)=6 n\left(3 n^{2}-1\right)+\left(6 n^{2}-6 n\right)\left(2 n^{2}-1\right)$. 
Some New Temperature Indices of Oxide and Honeycomb Networks

$$
\begin{aligned}
& T T\left(H C_{n}\right)=\frac{6 n}{3 n^{2}-1}+\frac{6 n^{2}-6 n}{2 n^{2}-1} . \\
& T Z\left(H C_{n}\right)=6 n\left(\frac{1}{3 n^{2}-1}\right)^{\frac{1}{2}}+\left(6 n^{2}-6 n\right)\left(\frac{1}{3 n^{2}-1}\right)^{\frac{1}{2}} . \\
& F T\left(H C_{n}\right)=6 n\left(\frac{1}{3 n^{2}-1}\right)^{3}+\left(6 n^{2}-6 n\right)\left(\frac{1}{2 n^{2}-1}\right)^{3} .
\end{aligned}
$$

Proof: Put $a=2,-2,1,-1,-1 / 2,3$ in equation (ii), we get the desired results.

\section{Conclusion}

In this paper, the first temperature index, modified first temperature index, temperature zeroth order index, $F$-temperature index, general first temperature index for oxide and honeycomb networks are computed.

Acknowledgement: The author is thankful to the referee for useful comments.

\section{REFERENCES}

1. V.R.Kulli, Multiplicative Connectivity Indices of Nanostructures, LAP LEMBERT Academic Publishing, (2018).

2. R.Todeschini and V.Consonni, Handbook of Molecular Descriptors for Chemoinformatics, Wiley-VCH, Weinheim, (2009).

3. V.R.Kulli, College Graph Theory, Vishwa International Publications, Gulbarga, India (2012).

4. S.Fajtlowicz, On conjectures of Graffiti-II, Congr. Numer.60(1987)187-197.

5. V.R.Kulli, Computation of some temperature indices of $H_{5} C_{5}[p, q]$ nanotubes, Annals of Pure and Applied Mathematics, 20(2) (2019) 69-74.

6. V.R.Kulli, The (a, b)-temperature index of H-Naphtalenic nanotubes, Annals of Pure and Applied Mathematics, 20(2) (2019) 85-90.

7. V.R.Kulli, Some multiplicative temperature indices of $H_{5} C_{7}[p, q]$ nanotubes, International Journal of Fuzzy Mathematical Archive, 17(2) (2019) 91-98.

8. V.R.Kulli, Multiplicative $(a, b)$-temperature index of H-Naphtalenic nanotubes, submitted.

9. T.K.Atework, P.N.Kisori and S.Dickson, Atom bond connectivity temperature index, Journal of Mathematical Nanoscience, 8(2) (2018) 67-75.

10. V.R.Kulli, Multiplicative connectivity $K V$ indices of dendrimers, Journal of Mathematics and Informatics, 15 (2019) 1-7.

11. V.R.Kulli, Multiplicative Gourava indices of armchair and zigzag polyhex nanotubes, Journal of Mathematics and Informatics, 17 (2019) 107-112.

12. V.R.Kulli, The $(a, b)$-status index of graphs, Annals of Pure and Applied Mathematics, 21(2) (2020) 113-118.

13. V.R.Kulli, Computation of multiplicative status Gourava indices of graphs, International Journal of Mathematical archive, 11(5) (2020) 23-28.

14. V.R.Kulli, Computation of status neighborhood indices of graphs, International Journal of Recent Scientific Research, 11(4) (2020) 38079-38085. 